\title{
Sex, gender and psychiatry: Is it important?
}

\author{
TN Rajapakse
}

There is an old joke about sex and gender, that goes like this: when asked to complete a routine form regarding personal data, in front of the box marked 'sex', the person filling the form innocently (and one assumes, truthfully), wrote, 'occasionally'.

Be that as it may, the terms sex and gender usually cause varying reactions, including, among others, curiosity, interest, dismissal or amusement, as shown by the previous example. But what of sex and gender in psychiatry: Is it worth our serious consideration?

\section{Influence of sex and gender on psychiatric illness}

When it comes to psychiatric disorders in general, there are many reported differences between males and females. Even if leaving aside the specific gender related disorders - reported rates of depression and bipolar affective disorder are greater in females; disorders such as autism, attention deficit hyperactivity disorder, alcohol use disorders and antisocial personality disorders are higher among males (1). Likewise with regards to suicide and self-harm behaviour - rates of suicide are greater in males compared to females the world-over, whereas rates of self-harm are greater among females; sometimes referred to as the 'gender paradox' of suicide (2). And these differences are not limited to numbers only. Schizophrenia is a striking example - although the gender prevalence of the illness is roughly equal, research shows that males tend to be more severely impaired in ratings of negative symptoms, whereas females have a better premorbid functioning and better prognosis (3). Similarly, the concept of a 'male depression' has been introduced, suggesting differences in the way males and females with depression may present (4).

Historically, differences in psychiatric illness among males and females have received much attention. And yet, our understanding of the true etiological and biological differences in psychiatric illness between males and females - if any - is still limited. Part of the challenge of doing research in this area is the difficulty of disentangling the role played by the person's biological sex, versus the effects of the person's gender: Sex is defined as the classification of a human being as male or female based on their chromosomal complement and reproductive organs; whereas gender is a person's self-representation as a man or a woman - a more social and cultural construct (5).
Sex differences in rates of illness between males and females are seen in many medical specialties; why focus on psychiatry in particular? Part of the answer is that in psychiatry, apart from biological sex, gender influences presentation much more than in other medical specialties. So for example - while it is generally accepted that depression is twice as common among females compared to males - is this due to a biological difference between the sexes? Or is it influenced by the different gender roles and cultural stereotypes assigned to males and females? Research has examined this question from multiple angles: proposed theories argue that it might be a true difference; or caused by females reporting more symptoms than males; or due to the fact that when patients present with the same symptoms, clinicians are more likely to diagnose depression in females compared to males (1). Thus the answer continues to be open to debate.

Similarly several theories have also been put forward to explain differences in the nature of schizophrenia in males compared to females. One explanation offered for the more prominent negative symptoms and poorer prognosis in males is the earlier age of onset, among males compared to females; and that oestrogen may be a protective factor which delays the age of onset of the disorder in females (6-7). Other researchers suggest that although it is currently grouped together as one illness, the schizophrenia marked by negative symptoms seen in young males, may be a 'different disorder' from the schizophrenia seen in older females, which is characterized by more affective symptoms, a better prognosis and a family history of affective disorders (8).

\section{Influence of sex and gender on the psy- chiatrist}

This is another way in which gender roles and attitudes influences psychiatry, more than in other specialties. Consider an example: If a female, who is known to have had several sexual relationships, is treated for appendicitis, the treating surgeon's disapproval of her 'morals' is unlikely to significantly impact the surgical treatment she receives. But if the same woman seeks help for depression from a psychiatrist, who looks down on her as a female of 'loose morals' (in conflict with the accepted female gender stereotype) - then, given the nature of the interactions between clinicians and patients in psychiatry - this is much more likely to adversely influence the therapeutic relationship and care she receives for depression. Thus, perceptions of gender 
roles and associated stereotypes have a greater likelihood of influencing clinician-patient interactions in psychiatry, compared to other specialties.

In the early twentieth century, psychiatrists (who were predominantly male) believed that females were more vulnerable to certain psychiatric disorders because they were the 'more emotional sex'; but to put this in context, this was at a time when females in the US were not allowed to vote, because it was believed that females were too emotional to make clear decisions as voters (9). Were the beliefs of clinicians at that time, influenced by the cultural perceptions of gender in that era? It seems very likely. We may now look back with amusement, at these assumptions regarding gender. And yet, I recognize that I myself am as entrenched within gender stereotypes of the present day, as were the clinicians of the past centuries; and while this is natural and to some degree inevitable, it is crucial to be aware of this. For instance, thanks to the cultural influence of gender roles: Are we paternalistic and more judgmental with regards to female patients, compared to males? Or in contrast, is more attention and care given to females who are perceived as 'weaker' - while the 'poor male' is ignored? This despite the evidence that suicide rates, for instance, are higher in males compared to females. It is fascinating to note that when examining the World Health Organization website on gender and mental health, the title of the page is actually, “Gender and Women's Mental Health" (10); what does this imply - does gender and mental health not include male mental health?

\section{Patient choice}

Research has also examined whether there are gender preferences among patients, regarding their choice of psychiatrist. A study from the United States reports that women constitute two-thirds of a female psychiatrist's patient load, compared to only one-half of a male psychiatrist's patient load (11). We do not have data regarding this for Sri Lanka; but given our own local cultural background, it would be interesting to research and explore whether clinician gender influences the patient's choice of a psychiatrist differently in Sri Lanka compared to the West.

\section{Implications}

Thus, sex and gender plays an important role in psychiatry. On one hand, both biological sex and gender roles influence patient presentations and the nature of psychiatric illness. As clinicians it is necessary to be aware of this, and recognize it where relevant - for example, the role that gender may play in the differences of clinical presentations of depression among males and females. In some disorders, such as in schizophrenia, while we recognize significant differences between males and females, our understanding of the etiology and implications for management between genders is still limited. Perhaps future research will give more definitive solutions to these questions, such as whether schizophrenia in young males versus in older females requires different classification and management.

And on the other hand, it is also equally important to be aware of, and self-monitor, how gender and gender attitudes may influence our own clinical practice in psychiatry. In the end, irrespective of gender, each patient is an individual human being, with their own background, biology, personality and culture - and deserves to be treated thus.

\section{Declaration of interest \\ None declared}

TN Rajapakse, Department of Psychiatry, Faculty of Medicine, University of Peradeniya.

Corresponding author: TN Rajapakse

E-mail: gemba471@gmail.com

\section{References}

1. Piccinelli M, Homen FG. Gender differences in the epidemiology of affective disorders and schizophrenia. Geneva: World Health Organization, 1997.

2. Canetto SS, Sakinfosky I. The gender paradox in suicide. Suicide Life Threat Behav1998; 28 (1): 1-23.

3. Shtasel DL, Gur RE, Gallacher F, Heimberg C, Gur RC. Gender differences in clinical expression of schizophrenia. Schizophr Res 1992; 7(3): 225-31.

4. Walinder J, Rutz W. Male depression and suicide. Int Clin Psychopharmacol 2001; 16: s21-24.

5. The Lancet Psychiatry. Sex and gender in psychiatry. Lancet Psychiatry 2016; 3(11): 999.

6. Ochoa S, Usall J, Cobo J, Labad X, Kulkarni J. Gender differences in schizophrenia and first-episode psychosis: a comprehensive literature review. Schizophrenia Res Treatment 2012; 2012: 916198.

7. Hafner H. Gender differences in schizophrenia. Psychoneuroendocrinology 2003; 28 Suppl 2: 17-54.

8. Castle DJ, Murray RM. The neurodevelopmental basis of sex differences in schizophrenia. Psychol Medicine 1991; 21(3): 565-75.

9. Hirshbein L. Sex and gender in psychiatry: a view from history. J Med Humanit 2010; 31(2): 155-70.

10. World Health Organization (WHO). Gender and Women's Mental Health. WHO, 2016 Available at: http:// www.who.int/mental_health/resources/gender/en/ (accessed 01 November, 2016).

11. Fenton WS, Robinowitz CB, Leaf PJ. Male and female psychiatrists and their patients. Am J Psychiatry 1987; 144(3): 358-61. 